\title{
Diseños metodológicos para la planificación participativa del paisaje
}

Methodological designs for participatory landscape planning

\author{
Jesús Oliva Serrano \\ Universidad Pública de Navarra \\ Andoni Iso Tinoco \\ Universidad Pública de Navarra \\ jesus.oliva@unavarra.es (ESPAÑA)
}

Recibido: 15.112012

Aceptado: 31.10 .2013

\section{RESUMEN}

Este trabajo analiza las metodologías participativas aplicadas al planeamiento y gestión del paisaje contrastando esta revisión con la experiencia desarrollada para la ordenación del monte San Cristóbal-Ezkaba en el área metropolitana de Pamplona-Iruñea (Navarra). Reflexionamos sobre sus posibilidades y ventajas, atendiendo de manera especial a las estrategias para recabar los sentidos y percepciones sociales del paisaje de diferentes grupos sociales e incorporarlas al proceso participativo. Nuestra investigación explora la efectividad de diferentes diseños metodológicos y técnicas para lograr este objetivo. Las peculiaridades de nuestro caso de estudio nos permiten profundizar en las dinámicas participativas y su potencial para animar un compromiso ciudadano con la conservación, planificación y gestión de los entornos cotidianos.

\section{PALABRAS CLAVE}

Convenio Europeo del Paisaje, planificación participativa, metodologías sociológicas 


\begin{abstract}
This paper analyse participatory methodologies applied to the planning and management of landscape and compares this analysis with the experience developed for the participatory planning of mount San Cristóbal-Ezkaba in the metropolitan area of Pamplona-Iruñea (Navarre). We reflect on its capabilities and advantages paying special attention to the strategies for gathering the social senses and landscape perceptions in different social groups and to incorporate them into the participatory process. Our research explores the effectiveness of different methodological designs and techniques to achieve this goal. The peculiarities of our case study allows us to examine the participatory processes and its potential to encourage citizen engagement with the conservation, planning and management of everyday environments.
\end{abstract}

\title{
KEYWORDS
}

European Landscape Convention, participatory planning, sociological methodologies

\section{INTRODUCCIÓN. PLANTEAMIENTO Y ESTRUCTURA DEL ARTÍCULO}

\subsection{Introducción}

Las metodologías para el planeamiento participativo del paisaje han experimentado un importante desarrollo. Se ha enriquecido con diseños sofisticados y una amplia innovación de las técnicas aplicadas. Si la planificación desde arriba sufría una progresiva deslegilimación social desde finales de los años 60, cuando Lefebvre (1969, 1971), Castells (1972) o Jacobs (1973) denuncian el urbanismo como ideología de la anti-ciudad, las concepciones clásicas del paisaje reducido a un espacio físico, neutro y objetivo, cuya ordenación requiere exclusivamente de los saberes especializados se replantea en las siguientes décadas (Gregory y Urry, 1985; Cosgrove, 1984, 1985). Frente a la tradicional visión estética, centrada en su dimensión ecológica, donde interviene el experto ordenando sus componentes naturales, los nuevos enfoques criticarán la dominación implícita en esa mirada y su primacía de lo visual (Scott, 2002). La propia concepción y representación del paisaje no deja de ser también un juego ideológico (Cosgrove y Daniels, 1988). Un producto, en definitiva, social y cultural. El interés por sus elementos morfológicos, ha dado paso al estudio del paisaje como experiencia social (Scott et al., 2009). Garantizar el compromiso ciudadano y la responsabilidad social con el paisaje se ha convertido en una estrategia fundamental para su 
protección. Pero entre las consecuencias del viejo planeamiento autocrático cabe reseñar la de haber consolidado un progresivo desinterés respecto a los proyectos para su ordenación y conservación.

\subsection{Objetivos, metodología y estructura}

Esta reformulación teórico-metodológica analiza las aspiraciones ciudadanas respecto al paisaje para incorporarlas a su planificación y gestión. Sin embargo, producir una información de esta naturaleza y garantizar una participación pública adecuada ha mostrado ser una tarea compleja. La ética política y la eficiencia metodológica aparecen necesariamente mezcladas en este campo. Por un lado, decidir quien y cómo participa así como la manera como será analizada su aportación define el proceso participativo en ambos aspectos. Por otro lado, es frecuente presuponer estas valoraciones como algo dado de antemano y que solo queda recabar rutinariamente con técnicas estandarizadas. En menos ocasiones se estudian los sentidos sociales del paisaje como experiencias compartidas, o como el producto de juegos reflexivos para consensuar propuestas y prioridades.

Nuestro trabajo, trata de contribuir a la clarificación de esta cuestión mediante una doble aproximación. Analizamos, por un lado, la metodología de mas de una veintena de casos relevantes de planificación participativa. Por otro lado, evaluamos la experiencia desarrollada para el plan de ordenación del monte San Cristóbal-Ezcaba en Pamplona-Iruñea (Navarra). Un diseño participativo dirigido por los autores a lo largo del 2010 para Paisatge, S.L y la Mancomunidad de la Comarca de Pamplona, mediante un contrato de investigación realizado con la Universidad Pública de Navarra. Un caso paradigmático para llevar a cabo nuestra indagación pues este monte que preside el norte del área metropolitana presenta una larga historia de fracasos para consensuar una ordenación integral que deriva en un alarmante abandono. La cima alberga además una inmensa fortificación subterránea reconvertida durante guerra civil y la postguerra en un duro penal cuya trágica historia, recientemente documentada, constituye hoy un referente de su imaginario social. Finalmente, como entorno ecológico integrado en la aglomeración urbana, el monte presenta un potencial de usos medioambientales, culturales y de ocio objeto de frecuentes y controvertidas polémicas ciudadanas.

Exploramos primero el paisaje como experiencia social. A continuación revisamos los modelos de planificación participativa empleados en diferentes ámbitos y su capacidad para integrar en estos procesos las percepciones sociales. Las fórmulas aplicadas por distintas iniciativas, programas y experiencias se resumen en la tercera sección. Después, contrastamos los hallazgos aprendidos en el desarrollo del proceso del PSIS del monte San Cristóbal-Ezkaba. En las conclusiones finales se resumen y discuten los principales resultados. 


\section{DE LA ESTÉTICA DEL PAISAJE A SU EXPERINCIA SOCIAL}

\subsection{Especies de espacios, lugares y paisaje}

Podemos interpretar nuestra relación con el espacio como una continua diferenciación de lugares. La historia humana puede leerse como una transferencia incesante de sentido social al entorno indiferenciado (lugares de enterramiento, enclaves sagrados, espacios públicos o privados, etc.). Para llevar a cabo este proceso, constituyente al fin y cabo de lo social, para vincularnos de una u otra forma a nuestros lugares, hemos recurrido a toda clase de estrategias. Desde las epifanías que designan la localización del futuro templo (Eliade, 1949) a los rituales fundadores de la ciudad (Ryckwert, 1976), la distribución de reliquias que designan lugares peregrinación (Zumthor, 1993) o representaciones imaginarias de la morada (Bachelard, 1957). Nos proyectamos así sobre nuestras las formas de organización espacial (Rapoport, 1977; Tuan, 1977) que pueden formar parte desde entonces de nuestra propia seguridad ontológica (Giddens, 1990). Como sugería Perec (1974), no hemos hecho otra cosa que multiplicar y diferenciar los espacios a nuestro alrededor.

Un elemento sustancial en esta semantización del territorio (García, 1976) ha sido sin duda la conceptualización del paisaje. Como expresaba Simmel, exige una integración de sus diferentes partes, "un ser-para-sí quizás óptico, quizás estético, quizá conforme al sentimiento, una exención singular y caracterizante» (1998: 176). Es decir, lo primero que cabe decir del paisaje es que se trata de algo que no existe sin el observador que otorga sentido al mismo (Bertrand, 2008) y que su información se halla encarnada en la propia sociedad (Caspersen, 2009). Surge como producto de un proceso sociocultural y su representación puede analizarse como ideología (Cosgrove, 1984, 1985) pero también como arquetipo profundo (Appleton, 1975, 1990).

El Convenio Europeo del Paisaje comienza definiendo como tal «cualquier parte del territorio tal como la percibe la población»(BOE, $05 / 02 / 2008$, art. 1.a) y demanda una implicación ciudadana en su interpretación y gestión, subrayando así la relación entre sociedad y entorno de la cual emana. Es el primer tratado con vocación de abarcar todos los paisajes (desde áreas naturales a espacios periurbanos) (Déjeant-Pons, 2006) y subraya los derechos y responsabilidades que todos tenemos en su protección, planeamiento y gestión. Pero también alberga las contradicciones latentes entre su concepción sociológica y el dominio dirigista oculto tras los discursos expertos. Como explica Olwig (2007), para el Convenio el paisaje «no es un ensamblaje dado de objetos físicos, que puede ser objetivamente analizado por el científico natural o social. Se trata más bien de una criatura de cambiante de identidades y percepciones culturales» (2007: 581). Por eso el informe explicativo aclara que las acciones no pueden reducirse 
ni monopolizarse por los aparatos técnicos y científicos. Sin embargo, este mismo informe, elaborado por un Comité de Expertos, solo propone medidas instrumentales (campañas informativas, de sensibilización, etc.) y formativas sobre los valores del paisaje previamente definidos por expertos. Se recupera así la idea estética y ecológica del paisaje, su concepción como panorama y la visión de arriba abajo (Olwig, 2007).

\subsection{Percepción y sentidos sociales del paisaje}

En tanto solo existe como relación y experiencia social, la interpretación del paisaje requiere explorar sus formas intersubjetivas. Para ello, es necesario expandir su práctica de la geografía a otras disciplinas y del experto al profano (Riesgo-Chueca y Gómez-Zotano, 2012). Su conceptualización a partir de los aspectos sociobiográficos, lejos de ofrecerse uniforme, suele presentar controversias que requieren una reflexión colaborativa. Como apunta Tilley (1994), «el espacio socialmente producido combina lo cognitivo, lo físico y lo emocional en algo que puede ser reproducido pero que está siempre abierto a la transformación y al cambio» (pág. 10). La complejidad que adquieren estas relaciones puede ilustrarse con el fenómeno del turismo rural (Oliva y Camarero, 2002, 2013). El paisaje es aquí un recurso principal para las economías locales y mercantilizar el rural charm, pues su imaginario social condensa las representaciones de lo natural, la identidad, la memoria, etc., revalorizadas por las economías de signos y lugares (Lash y Urry, 1990). Un elemento determinante tanto de la «autenticidad» buscada por el turista moderno (MacCannell, 1976) como de las performances aceptadas por el nuevo post-turista (Urry, 1990, 1995). Pero a medida que el turista se convierte en un personaje omnipresente (tomando fotos, preguntando, mirando, etc.) activa también una reflexividad local sobre los sentidos del paisaje. Como expresaba un joven agricultor del Pirineo entrevistado en otro trabajo de campo, «igual no sabemos apreciar lo que tenemos la gente de aquí; tanta naturaleza, lo bonito que es»(Oliva y Camarero, 2002: 24).

La propia demanda turística favorece una remodelación del paisaje como objeto de consumo de masas (Urry, 1995). Una agricultora encargada de un establecimiento de turismo rural entrevistada por García-Ramón (1995) indicaba que, cuando esperaba huéspedes, debía arreglar su entorno de manera que todo pareciera idílico, tal y como los turistas esperaban. No se trataba de mostrar su lugar de vida y trabajo cotidiano sino de preparar un «escenario» (podar arbustos, desbrozar senderos, limpiar el camino, etc.). El turista como flâneur postmoderno y el turismo como práctica generalizada suponen una transformación en la forma de experimentar el mundo que cambia también «sus apreciaciones estéticas de la naturaleza, el paisaje rural, el paisaje urbano y otras sociedades» (Lash y Urry, 1994: 256). 


\section{ESTRATEGIAS DE PLANIFICACIÓN PARTICIPATIVA DEL PAISAJE}

\subsection{Políticas y mecánicas participativas}

Las estrategias para superar el planeamiento autocrático, integrando la participación pública, han dado lugar a modelos y experiencias que pueden considerarse como un nuevo paradigma de planificación ascendente (cogestión, conservación comunitaria, desarrollo participativo, etc.). Las políticas que regulan el paisaje también han evolucionado hacia marcos legislativos más abiertos a la participación pública (consultas, derechos de petición, tribunales ciudadanos, foros, etc.). Por ejemplo, las metodologías aplicadas por las directivas de la Unión Europea para la política del agua (European Commission, 2002), evaluar el impacto medioambiental (2001/42/EC y la 2003/34/CE), el desarrollo rural (como el programa LEADER, basado propuestas de grupos locales) (European Commission, 2003) o la rehabilitación urbana (como el programa URBAN, que debe avalarse por foros participativos). El Convenio Europeo del Paisaje define la participación, en este sentido, como su instrumento básico de planificación y, autores como Prieur y Durousseau $(2003 ; 2006)$, interpretan esto como una extensión del Convenio de Aarhus, que promueve la democracia local en Europa. Otros programas internacionales del ámbito de la cooperación, el desarrollo rural (FAO, 2005) o la conservación medioambiental, aplican metodologías participativas de diagnóstico y gestión del paisaje (Ericson, 2006; Kalibo y Medley, 2007, Valencia-Sandoval et al. 2010). Por ejemplo, las auditorías Agenda 21 Local (ICLEI, 1996; FEMP, 1998).

En el marco de estas políticas se ensayan un surtido abanico de técnicas, desde «foto-cuestionarios» (Múgica y Delucio, 1996) a representaciones virtuales y visualización de escenarios (Tress and Tress, 2003; Lange and Hehl-Lange, 2005; Ericson, 2006); fotografías, transectos y paseos participativos (Scott et al. 2007); entrevistas adaptadas (Ericson, 2006; Höppner et al. 2007; Valencia-Sandoval et al. 2010) y talleres con agentes sociales (Waltz et al. 2007; Höppner et al. 2007; Valencia-Sandoval et al. 2010); cuestionarios específicos (Fernández, 2008) y discusiones de grupo (Höppner et al. 2007) o los mapeos comunitarios (Ericson, 2006; Kalibo y Medley, 2007).

La participación no puede ignorarse por unas administraciones que la necesitan tanto como la temen. Sus ventajas suelen verificarse en la legitimación del proyecto, la implicación y fomento de ciudadanías activas y el reforzamiento del compromiso público con su conservación (O’Rourke, 2005; Jones, 2007), etc. Sin embargo, como explica Scott (2002), «las constricciones de tiempo y recursos, junto con una reticencia a delegar las responsabilidad al público, han limitado el alcance e influencia de muchas participaciones a las estrategias preventivas convencionales» (pág. 2). Una contradicción que da lugar a evidentes abusos de las mecánicas participativas (Little, 1994; Luz, 2000; Cambell, 2001, Ericson, 2006). Por ejemplo, el estudio comparativo realizado por Prieur y Du- 
rousseau (2003) sobre la aplicación del Convenio Europeo muestra que la participación adquiere distintas interpretaciones en cada país. Desde aquellos que consideran como tal la participación de las propias administraciones públicas a los que estiman como protagonistas al individuo o agentes sociales interesados.

La participación requiere, por otro lado, un aprendizaje (Buchecker et al., 2003; Goodchild, 2006), mantener la transparencia del proceso y clarificar desde el inicio sus objetivos así como la influencia que tendrán los participantes en las decisiones finales. Las fórmulas que desvirtúan estas premisas van desde la mera manipulación a la participación pasiva o simplemente consultiva (Pretty, 1995) (como la participación sin información o la que guarda agendas ocultas de intereses). Estos abusos de las mecánicas participativas apenas consiguen garantizar el necesario compromiso ciudadano con el paisaje (Selman, 2004). Para que la sociedad se reapropie, como sugiere el Convenio Europeo, de sus responsabilidades con el paisaje, deben regenerarse con el proceso participativo las relaciones emocionales y simbólicas tanto como las medioambientales (Oliva e Iso, 2011, 2013). Por ejemplo, reconstruyendo sus valores intangibles (Nogué y Sala, 2006; 2008).

\subsection{Diseños metodológicos}

Nuestro estudio previo al diseño participativo supuso la revisión de más de una veintena de experiencias cuya principal conclusión fue la ausencia de un marco metodológico generalizado. El Cuadro 1 resume los diseños más relevantes. En general, han sido orientados a completar la identificación de unidades de paisaje por los actores sociales involucrados. Una cuestión frecuentemente planteada es cómo aunar los criterios técnicos-expertos con los elementos identificados por los participantes. A menudo es más una tarea de «traducción» para que las percepciones ciudadanas se expresen de forma técnicamente aceptable. Pero aunque intentan superar las lógicas meramente consultivas, muchas resultaban finalmente evaluaciones técnicas que, solo en fases posteriores, podrían ofrecer una participación en términos de Arnsteín (1969).

Este análisis permite resumir un proceso habitual en las fases genéricas siguientes:

1. Diagnóstico del paisaje/territorio. Inicialmente son análisis técnicos y sus herramientas son propias del paisajismo (definición de unidades sobre mapas, levantamientos 3D, geovisualizaciones, ortofotos, etc.) y pueden incluir estudios sociológicos para detectar opiniones de la población. La participación en esta primera fase es consultiva en la mayoría de las experiencias. Se difunde y contrasta la información con los actores sociales en presentaciones y jornadas con expertos, que preparan la fase siguiente de propuestas o escenarios. De forma excepcional, algunas experiencias involucran a los actores de forma más activa 
en el diagnóstico a través de talleres, petición de imágenes, itinerarios por el territorio, «técnicas de impacto" ", mapeos comunitarios, matrices históricas, etc.

2. Elaboración de propuestas y aprobación en un Plan. Es la fase que posibilita una mayor intervención pública pues define cómo se quiere el paisaje futuro y las medidas para lograrlo. Las herramientas tienen un carácter más abierto: talleres de futuro donde se definen posibles escenarios, planificación estratégica, árbol de problemas, role playing, jurados ciudadanos, talleres de priorización, preferencia visual, grupos de trabajo, foros, etc.

3. Gestión del plan. Este aspecto solo es contemplado en unos pocos casos y recae habitualmente en las administraciones con competencias en el territorio. Cuando se abre a la participación es a través de entidades o estructuras de partenariado que avalan la gestión del plan.

\section{Cuadro 1. Diseños metodológicos, agentes participantes y fases que implican}

\begin{tabular}{|c|c|c|c|}
\hline Zonas y objetivo & Diseño metodológico y técnicas & Participantes & $\begin{array}{c}\text { Fases de } \\
\text { participación }\end{array}$ \\
\hline $\begin{array}{l}\text { (Warren-Kretzsch- } \\
\text { mar et al., 2005) } \\
\text { Elm (Alemania) } \\
\text { Planificación del } \\
\text { paisaje }\end{array}$ & $\begin{array}{l}\text {-analisis «on-line» de propuestas de expertos } \\
\text {-plan disponible «on line» con posibilidad de } \\
\text { usar módulos, dibujar y comentar mapas y } \\
\text { borrador del texto } \\
\text {-seminarios, grupos de trabajo y excursio- } \\
\text { nes para temas relevantes (erosión del sue- } \\
\text { lo, renaturalización de una planicie, calidad } \\
\text { visual) }\end{array}$ & $\begin{array}{l}\text {-expertos } \\
\text { locales } \\
\text {-población } \\
\text { local }\end{array}$ & $\begin{array}{l}\text {-Diagnóstico } \\
\text {-Planificación }\end{array}$ \\
\hline $\begin{array}{l}\text { Rusdea, E. et al. } \\
\text { 2007) } \\
\text { Zona Montañas } \\
\text { Apuseni (Ruma- } \\
\text { nia) } \\
\text { Planificación } \\
\text { regional }\end{array}$ & $\begin{array}{l}\text {-entrevistas y cuestionarios a expertos, po- } \\
\text { líticos locales y agricultores para identificar } \\
\text { problemas } \\
\text {-síntesis en esquema de causas y relaciones } \\
\text {-»planning for real» con niños de escuela } \\
\text { local } \\
\text {-información y motivación mediante reunio- } \\
\text { nes, hojas informativas, conferencias, talle- } \\
\text { res, consultas, «role-playing», discusiones } \\
\text { de grupos } \\
\text { con especialistas, políticos y empresarios } \\
\text {-constitución de un Foro Comunitario con } \\
\text { científicos, autoridades, empresarios y ac- } \\
\text { tores clave para analizar tres escenarios de } \\
\text { tendencias futuras (tradicional, sostenible, } \\
\text { transformación) }\end{array}$ & $\begin{array}{l}\text {-responsables } \\
\text { locales } \\
\text {-expertos } \\
\text {-actores } \\
\text { sociales } \\
\text {-grupos de } \\
\text { interés }\end{array}$ & $\begin{array}{l}\text {-Diagnóstico } \\
\text {-Planificación } \\
\text {-Evaluación }\end{array}$ \\
\hline
\end{tabular}

${ }^{1}$ Cuyo objetivo no sólo es informar si no también generar un debate en la comunidad llamando la atención sobre el proceso (incluyen programas de televisión, exhibiciones, presentaciones con famosos, etc.) 


\begin{tabular}{|c|c|c|c|}
\hline Zonas y objetivo & Diseño metodológico y técnicas & Participantes & $\begin{array}{c}\text { Fases de } \\
\text { participación }\end{array}$ \\
\hline \begin{tabular}{|l|} 
Valencia-Sando- \\
val, C. et al., 2010) \\
Mèxico \\
Planificación del \\
paisaje
\end{tabular} & $\begin{array}{l}\text {-entrevistas semiestructuradas con agentes } \\
\text { clave } \\
\text {-mapeo en 3D para identificar tipos de suelo } \\
\text {-sintetización en un mapa base de diagnós- } \\
\text { tico } \\
\text {-taller para priorizar temas, identificar unida- } \\
\text { des del paisaje y recomendaciones } \\
\text {-visualizaciones y diagramas de síntesis }\end{array}$ & $\begin{array}{l}\text {-población } \\
\text { local } \\
\text {-promotores } \\
\text {-turistas } \\
\text {-propietarios } \\
\text {-construc- } \\
\text { tores } \\
\text {-agricultores } \\
\text {-trabajadores } \\
\text { del bosque } \\
\end{array}$ & $\begin{array}{l}\text {-Diagnóstico } \\
\text {-Planificación }\end{array}$ \\
\hline $\begin{array}{l}\text { Höppner et al. } \\
\text { 2007) } \\
\text { Zurich (Suiza) } \\
\text { Planificación del } \\
\text { paisaje }\end{array}$ & $\begin{array}{l}\text {-formación previa de Comité de expertos y } \\
\text { agentes sociales (agricultura, conservación } \\
\text { medioambiental, planeamiento, política lo- } \\
\text { cal) } \\
\text {-eventos informativos, taller, inspección so- } \\
\text { bre el terreno, encuentros, etc. }\end{array}$ & $\begin{array}{l}\text {-expertos } \\
\text {-agentes } \\
\text { sociales } \\
\text {-población } \\
\text { local } \\
\text {-políticos } \\
\text { locales } \\
\end{array}$ & $\begin{array}{l}\text {-Negociación } \\
\text {-Diagnóstico }\end{array}$ \\
\hline $\begin{array}{l}\text { (Walz, A. et al., } \\
\text { 2007) } \\
\text { Davos (Suiza) } \\
\text { Planeamiento } \\
\text { regional }\end{array}$ & $\begin{array}{l}\text {-talleres de expertos y agentes sociales } \\
\text {-formación de un Grupo Consejero } \\
\text {-ampliación a «grupos satélites» contactados } \\
\text { mediante técnica de «bola de nieve» } \\
\text {-talleres para sintetizar los procesos relevan- } \\
\text { tes (medioambientales, turísticos, agrarios, } \\
\text { etc.) }\end{array}$ & $\begin{array}{l}\text {-expertos, } \\
\text { agentes socia- } \\
\text { les y grupos } \\
\text { de interés } \\
\text { (política lo- } \\
\text { cal, turismo, } \\
\text { agricultura, } \\
\text { profesores, } \\
\text { pastores, etc.) }\end{array}$ & $\begin{array}{l}\text { Planificación } \\
\text { (ejes estraté- } \\
\text { gicos) }\end{array}$ \\
\hline $\begin{array}{l}\text { (Tress y Tress, } \\
\text { 2003) } \\
\text { Dinamarca } \\
\text { Planificación del } \\
\text { paisaje }\end{array}$ & $\begin{array}{l}\text {-análisis de } 4 \text { escenarios posibles de desa- } \\
\text { rrollo futuro mediante visualizaciones foto- } \\
\text { realistas (agricultura industrial recreación- } \\
\text { turismo, conservación natural, expansión } \\
\text { residencial) } \\
\text {-visualizaciones deliberadamente arquetípi- } \\
\text { cas (perspectivas aéreas y del suelo) presen- } \\
\text { tadas a grupos de agentes sociales y expertos } \\
\text {-cuestionario final para recabar su opiniones }\end{array}$ & $\begin{array}{l}\text {-políticos } \\
\text { locales } \\
\text {-técnicos de } \\
\text { planeamiento } \\
\text {-asociacio- } \\
\text { nes medioam- } \\
\text { bientales, } \\
\text { deportivas, de } \\
\text { cazadores } \\
\text {-agricultores }\end{array}$ & $\begin{array}{l}\text {-Planificación } \\
\text { (consulta de } \\
\text { escenarios) }\end{array}$ \\
\hline $\begin{array}{l}\text { (Kalibo y Medley, } \\
\text { 2007) } \\
\text { Monte Kasigau } \\
\text { (Kenia) } \\
\text { Gestión colabo- } \\
\text { rativa }\end{array}$ & $\begin{array}{l}\text {-mapeos del territorio con la población local } \\
\text { para obtener las visiones de género sobre el } \\
\text { monte y el bosque, identificar los elemen- } \\
\text { tos del paisaje, usos y distribución de sus } \\
\text { recursos } \\
\text {-reconstrucción de «mapas históricos» y un } \\
\text { «time-line» de eventos ocurridos en la zona } \\
\end{array}$ & $\begin{array}{l}\text {-población } \\
\text { local }\end{array}$ & -Diagnóstico \\
\hline $\begin{array}{l}\text { (Lange y Hehl- } \\
\text { Lange, 2005) } \\
\text { Zurich (Suiza) } \\
\text { Planificación del } \\
\text { paisaje }\end{array}$ & $\begin{array}{l}\text {-talleres con visualizaciones 3D en pantallas } \\
\text { con agentes sociales, expertos y los promo- } \\
\text { tores del proyecto }\end{array}$ & $\begin{array}{l}\text {-vecinos } \\
\text { residentes } \\
\text {-responsables } \\
\text { técnicos } \\
\text {-organizacio- } \\
\text { nes medioa- } \\
\text { mientales } \\
\text {-políticos } \\
\text { locales } \\
\end{array}$ & $\begin{array}{l}\text {-Planificación } \\
\text { (consulta) }\end{array}$ \\
\hline
\end{tabular}




\begin{tabular}{|c|c|c|c|}
\hline Zonas y objetivo & Diseño metodológico y técnicas & Participantes & $\begin{array}{c}\text { Fases de } \\
\text { participación }\end{array}$ \\
\hline $\begin{array}{l}\text { Roe, 2000) } \\
\text { Reino Unido } \\
\text { Planificación del } \\
\text { paisaje }\end{array}$ & $\begin{array}{l}\text { 1.reuniones para recabar propuestas } \\
\text { 2.consulta inicial para identificar temas } \\
\text { claves; } \\
\text { 3.informe descriptivo y definición de } \\
\text { objetivos; } \\
\text { 4.elaboración del documento y decisiones; } \\
\text { 5.implementación por un grupo de trabajo } \\
\text { constituido para cada proyecto }\end{array}$ & $\begin{array}{l}\text {-representates } \\
\text { locales } \\
\text {-grupos de } \\
\text { interes } \\
\text {-actores } \\
\text { sociales }\end{array}$ & $\begin{array}{l}\text {-Diagnóstico } \\
\text {-Planificación } \\
\text {-Gestión y } \\
\text { seguimiento } \\
\text { del Plan }\end{array}$ \\
\hline $\begin{array}{l}\text { Ericson, J.A. } \\
\text { (2006) } \\
\text { México } \\
\text { Planificación } \\
\text { territorial }\end{array}$ & $\begin{array}{l}\text {-mapeos comunitarios, matrices históri- } \\
\text { cas, diagramas institucionales, entrevistas } \\
\text { semiestructuradas, evaluación comunitaria } \\
\text { participativa }\end{array}$ & $\begin{array}{l}\text {-población } \\
\text { local }\end{array}$ & $\begin{array}{l}\text {-Diagnóstico } \\
\text {-Planificación } \\
\text {-Evaluación }\end{array}$ \\
\hline
\end{tabular}

Fuente: Elaboración propia

\subsection{Técnicas participativas y agentes sociales}

Los procesos estudiados identifican diferentes grupos de actores sociales (stakeholders) a los que se atribuye una importancia variable según sus competencias sobre el territorio, conocimientos o intereses directos (propietarios, vecinos, asociaciones, grupos profesionales, entidades que usan el territorio...). El Cuadro 2 resume los más habituales. Se trata de las administraciones públicas, sobre todo locales, que demandan iniciar un proceso de evaluación, planificación o análisis de impacto, contratando un equipo técnico para el desarrollo del proyecto. Según los casos, amplían la participación, por este orden, a otras entidades públicas, expertos, actores sociales (empresas, asociaciones, vecinos afectados...) y a la ciudadanía general. Los proyectos suelen considerar el paisaje como algo que concierne tanto a quienes tienen responsabilidades, como a propietarios o aquellos que pueden acceder y disfrutarlo (zonas limítrofes, turistas, deportistas, etc.).

\section{Cuadro 2. Actores habituales en el planeamiento participativo del paisaje}

\begin{tabular}{|l|l|}
\hline 1-Administración local. & $\begin{array}{l}\text { 4- Técnicos expertos en cuestiones de terri- } \\
\text { torio, paisaje y/o medio ambiente. }\end{array}$ \\
\hline $\begin{array}{l}\text { 2- Equipo técnico que realiza el proce- } \\
\text { so. }\end{array}$ & -Stakeholders. \\
\hline 3- Otras administraciones públicas. & 6- Ciudanía/público en general. \\
\hline
\end{tabular}

Para los diagnósticos suelen emplearse herramientas sociológicas usuales (encuestas, análisis de datos secundarios, entrevistas, talleres y discusiones de grupo) que, en sí mismas, no favorecen la participación, pues la mayoría de los 
proyectos consideran esta fase como técnica. La búsqueda de consensos desde el inicio del diagnóstico con todos los actores tampoco es la norma y solo se presenta en casos paradigmáticos. Los proyectos intentan una participación más orientada a unos grupos de actores que a otros según su relación con los objetivos y planes de actuación. Ciertas técnicas pueden dirigirse al público general pero otras suelen emplearse más con aquellos grupos definidos con una mayor responsabilidad o interés en el territorio (talleres, etc.). Para la participación general se activan otras herramientas más orientadas a la información y comunicación sobre el proceso que a la deliberación y decisión (jurados ciudadanos, técnicas de impacto...). En último término, la definición de quién y cómo participa no se explicita a priori en la mayoría de casos analizados, como tampoco sus límites y alcance de las propuestas (criterios de exclusión).

\subsection{Otros modelos de interés para nuestro estudio}

Algunas experiencias ofrecían un especial interés para nuestro proyecto, bien por su ámbito de actuación, la adecuación de las técnicas o la proximidad a nuestra realidad social o legislativa. Por ejemplo, el proceso realizado para el valle del río Irk (Reino Unido), surgido de la demanda de la Directiva de Aguas de la Unión Europea para planificar las cuencas hidrográficas. Promovido por el Ayuntamiento de Manchester, con la participación de agencias gubernamentales, organizaciones medioambientales, asociaciones de residentes y negocios se llevó a cabo dentro de un programa de doctorado de la Universidad de Manchester. Su objetivo fue elaborar un plan de sostenibilidad mediante escenarios de desarrollo sostenible. Fue elaborada una metodología para facilitar la participación en una planificación sostenible mediante su visualización en mapas conceptuales sobre las opciones posibles y sus consecuencias. Las once fases del proceso se basaron en talleres de corta duración a partir de los mapas. Las tres primeras correspondían al diagnóstico, reconocimiento del territorio y elementos técnicos. Las restantes fueron de planificación (límites, escenarios y propuestas), salvo la 8 que volvía a ser de diagnóstico (revisando los criterios y límites debatidos anteriormente). El proceso termina con la elaboración del plan y priorización de propuestas. No consta que se contemplase participación en la evaluación y gestión del mismo.

Los catálogos del paisaje de Cataluña constituyeron otra referencia fundamental. Concebidos normativamente ${ }^{2}$ como herramientas para la ordenación y gestión del paisaje, establecen ocho criterios entre los que destacan la multiplicidad de valores del paisaje atribuidos por los actores sociales, el enfoque cualitativo, su aplicabilidad al planeamiento territorial, la participación pública y la cooperación entre los agentes implicados (responsables políticos, expertos técnicos y científicos, ciudadanía, etc.). Su metodología puede resumirse en las fases de identificación y caracterización del paisaje, evaluación, definición de objetivos

${ }^{2}$ Ley 8/2005 de Protección, Gestión y Ordenación del Paisaje de Cataluña. 
de calidad paisajística, establecimiento de criterios y acciones. El diagnóstico se desarrolla en las dos primeras, que parten de un análisis técnico ambiental y de su percepción social (zonas más valoradas, itinerarios, valores emotivos, sentidos de pertenencia, etc.) que luego se contrasta con los actores mediante para realizar una evaluación DAFO del paisaje. No se plantean cuestiones acerca de la gestión posterior del plan. Las fases de planificación parten de una identificación de escenarios futuros deseables a partir de la información anterior buscando una implicación más amplia de la ciudadanía para definir objetivos y acciones.

Finalmente, la experiencia de planificación del paisaje de Yeste (Albacete) realizada por la Fundación +SUMA para este municipio empleaba consultas ciudadanas mediante análisis visuales de preferencias del paisaje realizadas por residentes y turistas para determinar las áreas de especial relevancia medioambiental. Con una gran riqueza cultural y natural, el entorno sufre la amenaza de la dispersión urbana mediante segundas residencias. La planificación, basada en modelos de vulnerabilidad y elementos de atracción integrados en un sistema de información geográfica, evaluaba las demandas y potencialidades de los usos del suelo así como los paisajes esperables en las décadas futuras, mediante técnicas de simulación y modelización digital del paisaje.

\section{DISEÑO PARTICIPATIVO PARA UN PAISAJE POLÉMICO}

\subsection{Peculiaridades de nuestro caso: el monte San Cristóbal-Ezkaba}

Con casi 900 metros y una superficie de 1.339 hectáreas, el monte San Cristóbal-Ezkaba constituye un referente paisajístico principal de la Cuenca de Pamplona-Iruñea; trasfondo visible de numerosas calles del centro y desde los pueblos metropolitanos. La cara sur, colindante con la aglomeración urbana, mantiene parte del bosque autóctono (encinas y robledales) en un paisaje históricamente modelado por los usos tradicionales (apicultura, pastoreo, canteras, viñedo, etc.). En la cima, un conjunto de antenas ofrece un impacto visual importante. Su vertiente norte, repoblada con pino, se abre en cambio a un valle rural y mantiene cotos de caza, explotaciones forestales y un acuartelamiento militar con áreas de entrenamiento. El monte conecta además con dos corredores ecológicos: una antigua vía férrea recuperada como sendero paisajístico que recorre el costado suroeste y un paseo fluvial que acompaña el Camino de Santiago al este.

Una larga historia de proyectos y propuestas fallidas debido a los distintos intereses de los siete ayuntamientos propietarios y administraciones implicadas en su gestión, ha impedido un planeamiento integral. La red informal de senderos formaba ya un entramado de más de 100 kilómetros y la proliferación de usos no regulados (escombreras, acceso con quads, etc.) y sucesivos incendios (en 1986, 2000 y 2005) completaban un panorama alarmante de abandono. Los municipios carecen de recursos para intervenir y las agencias regionales no logran consensuar unos programas globales de gestión (forestales, de riesgos, 
etc.). Además, algunas propuestas han sido muy contestadas (funicular a la cima, miniparque temático, etc.). Desde ya hace veinte años una asociación ciudadana reivindica mayor atención para el monte con una marcha anual a la cima (originalmente surgida para denunciar la presión urbanística). Otros colectivos (clubes de jubilados, asociaciones deportivas, escuelas taller, etc.) realizan eventos deportivos, jornadas de limpieza solidarias, repoblaciones, etc.

Pero la polémica que centra buena parte de las controversias es el futuro y la interpretación del Fuerte Alfonso XII, catalogado Bien de Interés Cultural. Un baluarte defensivo excavado en la cima que se hunde en varios pisos subterráneos, junto con un complejo de fosos de franqueo, estancias, aljibes, etc., abarcando más de 615.000 metros cuadrados. El dominio bélico de la aviación dejó obsoleta su función ya desde su finalización en 1919 y fue reconvertido, de 1934 a 1945, en un duro penal donde llegaron a hacinarse más de 2.000 prisioneros republicanos enterrados en sus galerías. En 1938 se produjo allí la mayor evasión de una prisión española fuertemente reprimida en los alrededores.

Algunos hechos solo se han conocido recientemente gracias al trabajo de historiadores y asociaciones, como la existencia de cementerios improvisados anexos. Desde entones el Fuerte es un referente de la reivindicaciones para la Memoria Histórica, que celebran los aniversarios de la fuga. La Sociedad Arantzadi ha identificado numerosos restos y se han devuelto más de 40 a sus familiares, contactados por la Asociación Txinparta. Diversos documentales (Alforja, 2005a; 2005b), biografías (Carratalá, 2007), etc., reseñan esta historia. La eliminación de ciertos elementos arquitectónicos, añadidos al convertirse en prisión, ha sido denunciada como un intento de reducir su interpretación a la obra de ingeniería militar (Autobús de la Memoria, 2001).

Además, su deterioro y accesibilidad hacen de este espacio un lugar continuamente visitado por curiosos y varias personas han tenido que ser rescatadas de su interior. El Ministerio de Defensa, propietario del Fuerte, ha planteado la necesidad de un convenio para su mantenimiento, mientras que algunos partidos políticos y ayuntamientos abogan por su cesión para gestionarlo como museo. Una opción sugerida también por la Ley de la Memoria Histórica que elabora el Parlamento Navarro.

\subsection{El proceso participativo}

Nuestro encargo fue diseñar un proceso participativo previo a la redacción del Plan por la Comisión responsable (compuesta por los municipios propietarios y técnicos de la Mancomunidad). El planteamiento original fue adaptar la metodología de Investigación-Acción-Participativa (Rodríguez-Villasante et al., 2000) al planeamiento del paisaje. Pero estas bases metodológicas, en su formulación ideal, habrían requerido una identificación previa de los actores participantes, elaborar un diagnóstico consensuado y concretar un plan de actuación (incluyendo la negociación de las estructuras de participación). Las condiciones del encargo, el presupuesto temporal y el marco regulador del Plan, limitaron 
el diseño a un proceso centrado en el diagnóstico, la identificación de unidades del paisaje y propuestas de usos para incorporar al PSIS. Esta experiencia logró consensuar criterios para una ordenación diferenciada según las áreas del monte, identificar las unidades fundamentales del paisaje y ordenar una red limitada de caminos abierta a distintas tipologías de usuarios. Pero también permitió explorar sus sentidos sociales y retroalimentar con esta información todo el proceso de participación y planeamiento.

De las cinco fases inicialmente definidas ${ }^{3}$ se ejecutaron tres (Cuadro 3). La primera daba a conocer el comienzo del proceso participativo y sus límites a través de un portal-web ${ }^{4}$ En la segunda fase, de diagnóstico, se realizaron 16 entrevistas a expertos, responsables políticos y técnicos relacionados con el monte y 5 grupos de discusión configurados con perfiles sociológicos seleccionados según variables generacionales, de género, localización residencial y usos del monte (ver Anexo). La información producida se incorporó al debate público y el proceso mediante diferentes estrategias. Por ejemplo, la polarización de discursos sobre el monte, sugerencias de intervención, etc., fueron sintetizadas en paneles, difundidas mediante el portal-web, como documentación para asociaciones y agentes sociales interesados, argumentario en los vídeos y presentaciones e informes técnicos para la Comisión del PSIS.

\section{Cuadro 3. Fases del proceso participativo}

\begin{tabular}{|l|l|l|}
\hline \multicolumn{1}{|c|}{$\begin{array}{c}\text { Fase 1. Difusión/ } \\
\text { Comunicación }\end{array}$} & \multicolumn{1}{|c|}{ Fase 2. Diagnóstico } & \multicolumn{1}{c|}{ Fase 3. Propuestas } \\
\hline -Entrevistas exploratorias & -Entrevistas a expertos. & -Taller 1 (Cara norte). \\
\hline $\begin{array}{l}\text {-Portal-Web (documentación, foro, } \\
\text { encuesta, mapa interactivo,...) }\end{array}$ & -Grupos de discusión. & -Taller 2 (agentes sociales). \\
\hline -Vídeos informativos & -Grupos de discusión. & \\
\hline & -Portal-Web (paneles) & \\
\hline
\end{tabular}

La tercera fase se organizó mediante Talleres con asociaciones y agentes sociales orientados a contrastar valoraciones y criterios de ordenación del monte. Una breve evaluación de las técnicas utilizadas muestra mejor su papel dentro del diseño metodológico.

${ }^{3}$ Fase I: Negociación de la propuesta de participación; Fase II: Diagnóstico; Fase III- Recogida de propuestas; Fase IV — Planificación (elaboración del PSIS); Fase V— Gestión y seguimiento.

${ }^{4}$ http://www.sancristobal-ezkaba.com/ En el momento de redacción del artículo se encuentra deshabilitado. 


\subsection{Valoración de las técnicas empleadas}

\subsubsection{Portal web}

En la fase inicial creó un cauce de comunicación general para difundir el proceso y más tarde abría espacios de debate y documentación del trabajo de campo (con secciones de Noticias, Información del Plan, Vídeos, Foro, Concurso de Fotos y Mapa Interactivo) fomentando la participación ciudadana en el debate, la posibilidad de aportar valoraciones, sugerencias e indicar elementos significativos del paisaje. El portal recibió más de 10.000. Por ejemplo, el Foro, con más de 4.000 visitas, recabó unas primeras reflexiones sobre el monte, representativas de participantes muy activos, visualizando cómo se articulaban los discursos más organizados (asociaciones ciclistas, de montaña, usuarios habituales, etc.) y permitiendo detectar de forma inmediata los problemas de comunicación (malentendidos, ideas falsas, etc.). Por su parte, el Concurso de Fotografía fue uno de los mayores revulsivos del portal-web. Formaba parte de la estrategia de comunicación, pero el análisis de las imágenes y comentarios ilustraba las representaciones arquetípicas atribuidas al paisaje. La propia idea del concurso implica ya una reflexividad sobre sus unidades y elementos significativos (tanto estéticos como afectivos). Es decir, aquellos considerados como relevantes para mostrar a los demás.

Otra herramienta de comunicación fueron los Vídeos, que ofrecen la posibilidad de visualizar a los «sin cara ni voz»o hacer un reconocimiento hacia los actores sociales del territorio. Se realizaron un total de tres ${ }^{5}$ difundidos a través del portal-web y Facebook. El primero documentaba la XVII Marcha Popular reivindicativa del monte organizada por el club Anaitasuna, con entrevistas a participantes y organizadores. El segundo mostraba una entrevista al presidente de la Mancomunidad explicando el concepto, implicaciones y fases del Plan. El último recogía, al modo de una memoria-informe, las propuestas de usos e itinerarios, percepciones, etc., trasladados a los responsables del Plan.

\subsubsection{Entrevistas y grupos de discusión}

Las entrevistas a expertos y responsables refieren discursos especializados sobre el monte y su problemática (ordenación del territorio, medioambiente, patrimonio cultural, agendas 21 locales, gestión forestal, bomberos, comandancia militar, etc.). Por ejemplo, sobre la necesidad de un Plan económicamente viable, de coordinar administraciones y agencias, de una gestión integral y agendas compartidas, de interpretar el fuerte como ingeniería emblemática, etc.). Unas perspectivas objetivadas por los sistemas conceptuales de disciplinas y especia-

${ }^{5}$ Disponibles en Youtube con los títulos «PSIS San Cristóbal Ezkaba», «XVII Marcha Popular San Cristóbal-Ezkaba Herri Martxa,» y «Parque Comarcal San Cristóbal-Ezkana Eskualde Parkea.» 
lidades profesionales. Frente a estos discursos expertos, los grupos de discusión ofrecen una apertura a los imaginarios sociales del monte y sus representaciones latentes en la experiencia social del paisaje. La sección siguiente analiza estas narrativas y su codificación ideológica. Tanto las entrevistas como los grupos de discusión proporcionaron argumentos decisivos para dinamizar el proceso participativo.

\subsubsection{Talleres con agentes sociales}

Los Talleres se organizaron como dos reflexiones colectivas sobre el proyecto y el paisaje. La primera aunaba elementos de diagnóstico y propuestas y fue realizada con representantes de diferentes sectores de la ladera norte, donde se mantienen usos rurales (forestales, caza, etc.). La intención inicial fue realizar un «transecto» participativo pero los presupuestos temporales hicieron necesario sustituirlo por una dinámica grupal consistente en detectar sobre plano los usos pasados, presentes y futuros. Esta información inspiró la propuesta final de itinerarios en esta ladera.

El segundo taller fue netamente propositivo. Realizado con 25 representantes enviados por asociaciones ciudadanas (culturales, medioambientales, de la Memoria, deportivas, de cazadores, etc.) interesadas en el proyecto y distribuidos en 5 mesas de trabajo configuradas de forma plural para confrontar diferentes visiones del monte en un diálogo sobre su ordenación y prioridades. Todos habían recibido previamente un dossier documental sobre el Plan, el proceso participativo realizado y resultados de trabajo de campo. Se pidió a cada grupo consensuar y concretar, sobre un mapa a escala, una propuesta de tres itinerarios justificando su adecuación, usuario al que se dirigía, etc. En la segunda parte del taller, cada portavoz explicaba las deliberaciones y propuestas de su mesa. Finalmente, se abría un debate general con intervenciones personalizadas y la participación de los técnicos de la Mancomunidad.

\section{EXPERIENCIAS Y ARQUETIPOS DEL PAISAJE}

Las representaciones del paisaje pueden analizarse como ideologías (Cosgrove, 1984, 1985), arquetipos (Appleton, 1975, 1990) y como experiencias sociales (Tuan, 1997; Tilley, 1994). Nuestras narrativas sobre el mismo, que incluyen todas estas formas, pueden sintetizarse también como tipologías discursivas (Soliva, 2007), como un aparato heurístico con el que enriquecer el proceso de planificación participativa. En nuestro caso, esta información fue elaborada a partir de los grupos de discusión realizados (ver Anexo). 


\subsection{Definiciones y representaciones del monte}

Las definiciones surgidas en los grupos plantearon una primera recomendación: considerar a lo largo de todo el proceso (comunicación, participación, etc.) las interelacciones emocionales, políticas y ecológicas. En la experiencia social del paisaje aparecen condensados los elementos sociobiográficos (infancia, identidad, etc.) (—es el monte que- «tengo identificado con mi niñez»-GD1-; «es mi primera vista todos los días» — desde mi habitación - GD2-»). Pero su definición también aparece frecuentemente asociada con su condición de espacio metropolitano (-es un monte que- «se va quedando como en el medio de la ciudad»-GD1-;»es el pulmón (...) que tenemos ahí»-GD2-). Otras categorizaciones definen el monte por su problemática política («es el monte peor gestionado de la Cuenca»-GD2_; «es un gran desconocido» — no se interpreta, no se informa- GD2-; — es un monte con-»intereses creados»-con una interpretación problemática - GD3-).

Estas percepciones permitieron sintetizar algunos planteamientos de partida para el resto del proceso participativo. Por ejemplo, una ciudadanía progresivamente distanciada de la producción del paisaje mostraba visiones idealizadas de «lo natural» que rechazaban cualquier intervención («no tocar»; «dejarlo como está»). El paisaje se concibe aquí como resultado espontáneo de una naturaleza inmaculada («me gusta que no se toquen las cosas, me parecen más naturales» (G5: mujer, 34 años). Frente a esta narrativa, aquellos participantes más conscientes de la elaboración socio-histórica del paisaje (mayores con un pasado rural, profesionales agrarios...) destacaban la necesidad de intervenir para preservarla («el monte tienes que adecentar (...) para que se pueda mantener, porque sino se deteriora» - G5: mujer, 59 años-). También aquellos más cotidianamente vinculados al monte (deportistas, vecinos, etc.) mostraban un mayor reconocimiento del entorno (zonas, senderos, usos, etc.) y concreción en sus propuestas (gestionar la accesibilidad, ordenar los caminos, etc.) mientras el resto orientaba más su opinión hacia las cuestiones generales.

La constelación de sentidos y representaciones del monte ofrecía así una tipología de estructuras discursivas que guardan patrones perceptivos arquetípicos sobre sus problemas, prioridades y soluciones. Por ejemplo, un discurso conservacionista advertía del peligro de devaluar el paisaje y priorizaba su regeneración (incluso desde posiciones sesgadas por su condición de usuario para reclamar un monte «preparado y listo para usar» - G2: varón, 33 años, usuario deportivo-). Una visión recurrente también en el Foro-web que se fue matizando al contrastar con el resto. Otra tipología encarnaba un discurso posibilista abogando sin contradicción por intervenir para conservar el monte y adecuación al disfrute general («lo queremos un monte, pero arreglado»-GD2, mujer, 37 años-). La necesidad de interpretar el monte en relación con la historia del penal aparecía sintetizada por un discurso de la Memoria. Aquí los sentidos esenciales del paisaje se unían a la interpretación (educativa, museística, etc.) del Fuerte y la demanda de respeto (no comercializar o convertirlo en atracción turística) («que cuente la historia del fuerte en sí»—GD3-). Finalmente, un 
discurso desconfiado, mucho más difuso, esgrimía una crítica generalizada ante cualquier propuesta ( ¿inos interesa? - G2: mujer, 52 años-) evidenciando el vacío comunicativo sobre el Plan previo a nuestro trabajo de campo.

\subsection{Regulación del paisaje y derechos simbólicos}

Nuestra experiencia sugiere también claves para reforzar el compromiso ciudadano con el paisaje. La ampliación del ámbito de interacción que favorece la difusión del automóvil relega muchos entornos en las sociedades modernas a la condición de intersticios metropolitanos ( «yo antes si que -iba al San Cristóbal- (...) pero ahora ya me voy a otras zonas» —G1: varón, 27 años-). La responsabilidad que manteníamos con estos paisajes perdura sin embargo en el recuerdo de las generaciones maduras («era toda la mañana del domingo, ibas allá» (G4); «me recuerda mi niñez, con mi abuelo (...) siempre íbamos a andar» (G2: mujer, 34 años). Esta conciencia del distanciamiento que experimentamos se presentaba con unas formas de codificación que pueden aplicarse eficazmente en las estrategias (comunicativas, participativas, etc.) para involucrar a la ciudadanía en su reapropiación ( «hay mucha gente que no ve San Cristóbal como un monte en sí» -G3: mujer, 19 años-; «al quedar tan cerca de Pamplona es como si van a un parque - del centro urbano-» - G3: varón, 25 años-).

Las argumentaciones relacionadas con unos supuestos derechos prioritarios sobre el monte de unos grupos frente a otros, también ofrecieron un material decisivo para ordenar la reflexión participativa sobre el monte y su planeamiento. Estas distinciones afloraban en el debate sobre la regulación de la accesibilidad (mejorar o no los accesos, facilitar o no un transporte público a la cima, etc.), evocando perfiles de buenos y malos usuarios, como los habituales (ciclistas deportivos) o auténticos (montañeros, etc.) frente a los domingueros («el monte es de todos pero (...) debería ser un poco de quien lo usa»-GD1-; "parece que son los únicos que saben ir al monte - los montañeros sobrados-, o los únicos que tienen derecho»-GD3-); los vecinos colindantes frente al resto; el turista frente al local, etc., polarizando las posiciones discursivas. Por ejemplo, el grupo de mujeres proponía garantizar una accesibilidad generalizada (con mayores, niños, dependientes, etc.) (»hay mucha gente que no puede subir más que en coche»-G5: mujer, 59 años-; «arreglarlo para todos»-G5: mujer, 35 años-) que contrastaba la visión de usuarios deportivos habituales (jóvenes, varones, deportistas) que pedían un acceso restringido («el monte es para ir al monte» -G1: varón, usuario deportivo-). Uno de los paneles difundidos para dinamizar el debate y la comunicación del proceso sintetizaba ambas posiciones con citas literales.

Pero además, en este debate se identifican ya las unidades principales del paisaje finalmente consideradas para el Plan. Por ejemplo, la cima condensaba todas las significaciones, pues el tipo de actuación allí (como el tratamiento otorgado a su accesibilidad o al Fuerte) condicionaba el monte en su conjunto («si ponen un restaurante ahí arriba, pues cambiaría todo»-GD4-; «cambia el 
concepto de monte (....) Ya no podrías utilizarlo como monte»-GD1-). La controversia se debilitaba al tratar la media ladera sur, intocable para los usuarios deportivos pero que debía ofrecer mayor accesibilidad para el resto («tendría que buscarse un equilibrio ¿no? -GD4-). Y finalmente desaparecía en relación con la falda sur colindante a la ciudad (donde el consenso para ordenarla como área de esparcimiento general —paseos, instalaciones deportivas al aire libre, etc.- era completa) y la cara norte (valorada más directamente con la estética del paisaje ecológico, que definía el monte-monte, y una intervención blanda para usos especializados —-senderismo, bicicleta, etc.-).

\section{CONCLUSIONES}

Las cuestiones implícitas en el planeamiento participativo del paisaje ofrecen, como vemos, una doble problemática política y metodológica. El análisis realizado muestra cómo aparecen relacionadas en los presupuestos teórico-metodológicos, en los diseños y las experiencias concretas. Por un lado, los objetivos de la planificación ascendente pueden ser subvertidos o reforzados por las propias formas de aplicación de estas metodologías. Además, la experiencia social del paisaje y su interpretación no se ofrecen de una manera lineal y el empleo de una herramienta participativa, por sí misma, no supone participación real. La percepción social de ciertos grupos es frecuentemente invisibilizada, bien por propios los propios diseños, bien por el análisis que se hace de su información.

En nuestro caso, por ejemplo, pudimos constatar una subrepresentación femenina en todos los ámbitos. Desde los miembros de la comisión responsable del Plan a los técnicos, desde los expertos entrevistados a los representantes enviados por las asociaciones al segundo Taller (apenas 2 mujeres de los 25 participantes). Esta relación social, que aparece continuamente reflejada en los procesos participativos, excluye una experiencia social del paisaje estratégica para su interpretación. La previsión de esta deriva sociológica en nuestra experiencia trato de abordarse con una presencia equilibrada por género en los grupos de discusión y la configuración de otro exclusivamente de mujeres. Las aportaciones de este último fueron decisivas para establecer los criterios de ordenación del monte y contrastar su experiencia social del paisaje con las de otros perfiles que tienden a dominar la mecánica institucional y participativa del proceso. Pero esto, solo fue posible, gracias al tratamiento finalmente otorgado a su información.

Por otro lado, en la regulación y las políticas orientadas al planeamiento participativo del paisaje subyacen aun las viejas tensiones entre las visiones ecológicas y su conceptualización como producto social, entre el dirigismo experto y la necesidad de involucrar a la ciudadanía en su compromiso con el paisaje. Nuestro análisis evidencia la inexistencia de un modelo metodológico generalizado que supere este conflicto y sugiere la importancia de concebir diseños donde la triangulación de técnicas y perfiles participantes permita profundizar en los aspectos emocionales y sociobiográficos que definen el paisaje como expe- 
riencia social. Una información, como hemos tratado de mostrar, revela claves de codificación ideológica, arquetipos y formas tipológicas de conceptualización de problemas y soluciones especialmente útiles para definir el planeamiento y promover la reapropiación ciudadana de sus derechos y responsabilidades con el paisaje.

\section{BIBLIOGRAFIA}

ALFORJA, I.

(2005a): Ezkaba, la gran fuga de las cárceles franquistas. Eguzki Bideoak (documental)

(2005b): El cementerio de las botellas. Eguzki Bideoak (documental)

APPLETON, J.

(1975): The Experience of Landscape. London, Wiley Press.

(1990): The Symbolism of Habitat. Washington, University of Washington

ARNSTEIN, S.R. (1969): «A Ladder of Citizen Participation», Journal of the American Planning

Association, 35 (4): 216-224.

AUTOBÚS DE LA MEMORIA (2001): San Cristóbal-Ezkaba: muros derribados para amurallar la memoria. (http:/www.autobúsdelamemoria.org) 1965

-BACHELARD, G. (1957): La poética del espacio. Buenos Aires, FCE,

BERTRAND, G. (2008): «Un paisaje más profundo. De la epistemología al método», Cuadernos Geográficos, 43: 17-27

BORSOTTO, P.; HENKE, R.; MACRI, M.C y SALVIONI, C. (2008): «Participation in Rural Landscape Conservation Schemes in Italy», Landscape Research, 33(3): 347-363

BUCHECKER, M.; HUNZIKER, M y KIENEST, M. (2003): «Participatory landscape development: Overcoming social barriers to public involvement». Landscape and Urban Planning, 64: 29-46.

CAMPBELL, J. R. (2000): «Participatory rural appraisal as qualitative research: distinguising methodological issues from participatory claims». Human Organization; J. Soc. Appl. Anthrop. 60(4): 380-389 Pamiela

CARRATALÁ, E. (2007): Memorias de un piojo republicano. Pamplona.

CASPERSEN, O.H. (2009): «Public participation in strengthening cultural heritage: The role of landscape character assessment in Denmark». Geografisk Tidsskrift-Danish Journal of Geography, 109 (1): 33-45

CASTELLS, M. (1972): La cuestión urbana. Madrid, Siglo XXI, 1974 COSGROVE, D.

(1984). Social Formation and Symbolic Landscape. London: Croom Helm

(1985): «Prospect, perspective and the evolution of the landscape idea», en Transactions of the Institute of British Geographers, 10(1): 45-62. 
COSGROVE, D and DANIELS, D. (1988) (eds.): The Iconography of Landscape. Essays on the Symbolic Representation, Design and Use of Past Environments. Cambridge, Cambridge University Press

DÉJEANT-PONS, M. (2006): «The European Landscape Convention», in Landscape Research, vol. 31(4): 363-384

-ERICSON, J.A. (2006): «A participatory approach to conservation in the Calakmul Biosphere Reserve, Campeche, Mexico», Landscape and Urban Planning, 74: 242-252

EUROPEAN COMMISSION (2002): Guindance on public participation in relation to the water framework directive. Brussels. European Commission.

EUROPEAN COMMISSION (2003): Rural Development in the European Union. Fact Sheet, Brussels. European Commission.

EUROPEAN LANDSCAPE CONVENTION (2000): European treaty series, 176. Council of Europe.

EUROPEAN SPATIAL PLANNING and LANDSCAPE, números 74, 72, $82,83,84,88,89,90,91$ que recogen los 9 Meetings of the Workshops for the implementation fo the European Landscape Convention (de 2002 a 2010).

FAO (2005): An Approach to rural development. Participatory and Negotiated Territorial Development. Rural Development Division

FEMP (Federación de Municipios y Provincias) (1998): Código de Buenas Prácticas Ambientales. Madrid. Federación de Municipios y Provincias

FERNÁNDEZ, $\mathrm{S}$.

(2008). «Participación pública, gobierno del territorio y paisaje en la Comunidad de Madrid», en Boletín de la Asociacion de Geografos Españoles, 46: 97-119.

GARCÍA, J.L. (1976): Antropología del territorio. Madrid, Ediciones B

GARCÍA-RAMÓN, M.D. (1995): «Trabajo de la mujer, turismo rural y percepción del entorno: una comparación entre Cataluña y Galicia», en Agricultura y Sociedad, 75: 115-152.

GIDDENS, A. (1990): Consecuencias de la modernidad. Madrid, Alianza, 1993.

GOODCHILD, P.H. (2006): «The skills of training the public for participation in decision-making processes». European Spatial Planning and Landscape, 83: 211-225.

GREGORY, D. y URRY, J. (eds) (1985): Social Relations and Spatial Structures. Londres, Macmillan

HÖPPNER, C.; Frick, J. and Buchecker, M. (2007): «Assesing psycho-social effects of participatory landscape planning». Landscape and Urban Planning, 83: 196-207.

ICLEI (Local Goverments for Sustainability) (1996): Local Agenda 21 Planning Guide. (Http:/www.iclei.org) (06/21/2012)

JACOBS, B. (1973): Muerte y vida de las grandes ciudades. Madrid, Península.

JONES, M. (2007): «The European Landscape Convention and the question of public participation». Landscape Research, 32(5): 613-633 
KALIBO, H.W, MEDLEY, K.E. (2007): «Participatory resource mapping for adaptive collaborative, management at Mt. Kasigau, Kenya». Landscape and Urban Planning, 82: 145-158

LANGE, E. y HEHL-LANGE, S. (2005): «Combining a Participatory Planning Approach with a Virtual Landscape Model for the Siting of Wind Turbines». Journal of Environmental Planning and Management, 48(6): 833-852. ledge

LASH, S. y URRY, J. (1994): Economies of signs and space. Londres, Rout-

LEFEBVRE, $\mathrm{H}$.

(1969). El derecho a la ciudad. Barcelona, Península.

(1971): De lo rural a lo urbano. Barcelona, Península.

LITTLE, P.D. (1994): «The link between local participation and improved conservation. A review of issues and experiences» en Western, D.; Wright, R.M. (Eds.), Natural Connections: Perspectives in Community-based Conservation. Island Press, Washington, D. C., pp. 347-372.

LUZ, F. (2000): «Participatory landscape ecology. A basis for acceptance and implementation». Landscape and Urban Planning, 50: 157-166

MACCANNELL, D. (1976): The Tourist. A New Theory of the Leisure Class. Berkley, University of California Press, 1999

MÚGICA, M and DELUCIO, J. (1996): «The role of on-site experience on landscape preferences. A case study at Doñana National Park, Spain», in Journal of Environmental Mangement, 47: 229-239.

-NOGUÉ, J.; Sala, P.

(2006): «The Spanish experience: landscape catalogues and landscape guidelines of Calatonia». European Spatial Planning and Landscape, 84: 121-145

(2008): «El paisaje en la ordenacion del territorio, los catálogos de paisaje en Cataluña», en Cuadernos Geográficos, 43: 69-98

OLIVA, J and CAMARERO, L.

(2002): Paisajes sociales y metáforas del lugar: una exploración de la ruralidad itinerante en Navarra. Pamplona, Universidad Pública de Navarra

(2013): «Touristic Processes, 'Generic Rurality' and Local Development», in Figueiredo, E. and Raschi, A. Fertile Links? Connections between tourism activities, socioeconomic contexts and local development in European rural areas. Florence, Firenze University Press

OLIVA, J. e ISO, A.

(2011): «Participatory methodologies in landscape planning», ponencia presentada al Colloquium Methodological Challenges in Participatory Research, IESA-CSIC, Córdoba

(2013): «Planeamiento urbano, participación y paisaje. Comparación de dos experiencias en la Cuenca de Pamplona-Iruñea», en XI Congreso Español de Sociología, Madrid, Actas del Congreso.

OLWIG, K.R. (2007): «The Practice of Landscape 'Conventions' and the Just Landscape: The Case of the European Landscape Convention», in Landscape Research, 32 (5): 579-594 
O'ROURKE, E. (2005): «Landscape planning and community participation: Local lessons from Mullaghmore, the Burren National Park, Ireland». Landscape Research, 30(4): 483-500

PRETTY, J.N. (1995): «Participatory learning for sustainable agriculture». World Development, 23(8): 1247-1263

PEREC, G. (1974): Especies de espacios. Barcelona, Montesinos

PRIEUR, M., Durousseau, S.

(2003): Comparative study of legislation on public participation in landscape matters in the context of implementation of the European Landscape Convention. European Council, Strasburgo, TFLOR

(2006): Landscape and Sustainable Development. Challenges of the European Landscape Convention. European Council, Strasburgo

RAPOPORT, A. (1977): Human aspects of urban form. Oxford, Pergamon

RIESGO-CHUECA, P y GÓMEZ-ZOTANO, J. (2012): «Landscape Fieldwork: Scientific, Educational and Awareness-Raising Requeriments in the Context of the European Landscape Convention», in Landscape Research, págs 1-12

RODRÍGUEZ VILLASANTE, T.R., MONTAÑÉS, M. y MARTÍ, J. (2000): La investigación social participativa. Barcelona,Viejo Topo

ROE, M. (2000): «Landscape Planning for Sustainability: community participation in Estuary Management Plans», Landscape Research, 25(2): 157-181

RUSDEA, E.; Reif, A.; Konold, W y Rotar, I. (2007): «An inter and transdisciplinary approach to support sustainable land-use. A case study in the Apuseni Mountains in Romania», en European Spatial Planning and Landscape, 88: 341-348.

RYCKWERT, J. (1976): La idea de ciudad. Salamanca, Ediciones Sígueme, 2002

Scott, A.

(2002): «Assessing Public Perception of Landscape. The LANDMAP experience», en Landscape Research, 27 (3): 271-295

SCOTT, A.; CARTER, C.; BROWN, K. and WHITE, V. (2009): «Seeing is Not verithing': Exploring the Landscape Experiences of Different Publics», in Landscape Research, 34(4): 397-424.

SELMAN, P. (2004): «Community participation in the planning and management of cultural landscape». Journal of Environmental Planning and Management, 47 (3): 365-392

SIMMEL, G. (1998): «Filosofía del paisaje», en El individuo y la libertad. Ensayos de crítica de la cultura. Madrid, Península, págs. 175-186 (e.o.1913)

SOLIVA, R. (2007): «Ladscape stories: Using idel type narratives as a heuristic device in rural studies», en Journal of Rural Studies, 23: 62-74.

TILLEY, CH. (1994): A Phenomenology of Landscape. Oxford, Berg.

TRES, B. and TRESS, G. (2003): «Scenario visualisation for participatory landscape planning —a study from Denmark». Landscape and Urban Planning, 64: 161-178.

TUAN, Y. (1977): Space and Place. The Perspective of Experience. Minneapolis, University of Minnesota Press (2001). 
URRY, J.

(1990): The Tourist Gaze: Leisure and Travel in Contemporary Society. Sage, London

(1995): Consuming Places. Routledge, London

VALENCIA-SANDOVAL, C., FLANDERS, D.N y KOZAK, R.A. (2010): «Participatory landscape planning and sustainable community development: Methological observations from a case study in rural Mexico». Landscape and Urban Planning, 94: 63-70.

WALZ, A.; LARDELLI, C.; BEHRENDT, H.; GRET-REGAMEY, A.; LUNDSTRÖM, C.; KYTZIA, S. y BEBI, P.( 2007): Participatory scenario analysis for integrated regional modelling. Landscape and Urban Planning, 81: 114-131.

WARREN-KRETZSCHMAR, B.; NEUMMAN, A. y MEIFORTH, J. (2005): «Interactive Landscape Planning. Results of a pilot study in Koenigslutter am Elm, Germany». Proceedings CORP

ZUMTHOR, P. (1993): La medida del mundo. La representación del espacio en la Edad Media. Madrid, Cátedra, 1994

\section{ANEXO \\ CONFIGURACIÓN DE LOS GRUPOS DE DISCUSIÓN}

El diseño original de los grupos de discusión trataba de combinar las variables de género, generacionales, localización residencial en el área metropolitana y usos habituales del monte. Las sesiones fueron celebradas en la Universidad Pública de Navarra y dirigidas por los autores. La contactación y gratificación fue realizada por una empresa externa de apoyo a los trabajos de campo. Su configuración final fue la siguiente:

GRUPO 1. JÓVENES COLINDANTES (19/10/2010). Configurado por jóvenes residentes durante los últimos cinco años en distritos de Pamplona y municipios metropolitanos colindantes al monte, buscando un cierto equilibrio de sexos y lugares de residencia y con al menos un participante usuario habitual de este espacio en su orientación recreativa, deportiva o naturalística. Compuesto finalmente con 7 participantes (4 varones y 3 mujeres) de 24 a 29 años procedentes de Berrioplano, Chantrea, Villava, Ansoain y Berriozar. La discusión evoluciona desde las cuestiones de la accesibilidad al monte y su regulación (para evitar la masificación o usos no deseados) a los derechos simbólicos sobre el monte y la desconfianza frente a los proyectos institucionales, el peligro de comercializar el monte y la necesidad de darle un uso equilibrado.

GRUPO 2. JÓVENES NO COLINDANTES (27/10/2010). Configurado por jóvenes residentes durante los últimos cinco años en distritos y pueblos metropolitanos no colindantes al monte y buscando un equilibrio de sexos y lugares de 
residencia además de la participación de al menos un usuario habitual del espacio. Compuesto finalmente con 8 participantes (5 mujeres y 3 varones) de 19 a 28 años residentes en Burlada, Iturrama, Milagrosa, San Jorge, Barañain, Huarte y Segundo Ensanche. La discusión evoluciona desde la cuestión del fuerte y la necesidad de interpretarlo en relación con la memoria histórica a la desconfianza de la política y los proyectos en general; la necesidad de conservar y cuidar el monte; de prevenir los comportamientos inadecuados y el abandono, y sobre su condición de monte frente a sus usos como parque urbano.

GRUPO 3. VECINOS COLINDANTES MADUROS (20/10/2010). Configurado por residentes durante los últimos cinco años en municipios o distritos de Pamplona colindantes al monte y buscando un equilibrio por sexo, grandes grupos de edad y lugares de residencia así como al menos un participante usuario habitual. Compuesto finalmente con 8 participantes (4 mujeres y 4 varones) de entre 34 y 52 años procedentes de Rochapea, Villava, NuevoArtica, Berriozar y Chantrea). La discusión evoluciona desde la necesidad de adecuar el monte o bien dejarlo como está; cómo adecuar los caminos; sus usos y posibilidades a la cuestión del tratamiento del fuerte y la memoria histórica.

GRUPO 4. VARONES MADUROS NO COLINDANTES (13/10/2010): Configurado por varones residentes durante los últimos cinco años en municipios o distritos no colindantes al monte y buscando un equilibrio de grandes grupos de edad y lugares de residencia así como la participación de al menos un usuario habitual del monte. Compuesto finalmente con 8 participantes de entre 32 y 53 años procedentes de Milagrosa, Ermitagaña, Mutilva, Casco Viejo, Burlada, Huarte y Barañain. La discusión se desenvuelve en torno a los ejes relacionados con la necesidad de acondicionar o no la carretera de acceso principal; el peligro de masificación turística; las diferencias de acceso entre los distritos colindantes y el resto; las diferencias entre las caras norte y sur; los usos deportivos especializados y el tipo de actuación para el fuerte y la cima.

GRUPO 5. MUJERES MADURAS NO COLINDANTES (26/10/2010). Configurado por mujeres residentes durante los últimos cinco años en municipios o distritos del área metropolitana no colindantes al monte y buscando un equilibrio según grandes grupos de edad y lugar de residencia así como al menos un participante usuario habitual del monte. Compuesto finalmente con 8 participantes de entre 35 y 60 años de edad residentes en San Juan, Mendillorri, Villava, CascoViejo, Sarriguren, Ermitagaña y San Jorge. La discusión evoluciona en relación con el abandono del fuerte y la inseguridad de un monte tan cercano a la ciudad; las limitaciones de accesibilidad y uso para ciertos grupos; la necesidad de recuperarlo y cuidarlo y sobre sus posibilidades en relación con los usos educativos y recreativos. 